\title{
Landau damping of Gardner solitons in a dusty bi-ion plasma
}

\author{
A. P. Misra ${ }^{1, \text { * }}$ and Arnab Barman ${ }^{1}$ \\ ${ }^{1}$ Department of Mathematics, Siksha Bhavana, Visva-Bharati University, Santiniketan-731 235, West Bengal, India
}

(Received 31 March 2015; Revised 10 June 2015)

\begin{abstract}
The effects of linear Landau damping on the nonlinear propagation of dust-acoustic solitary waves (DASWs) are studied in a collisionless unmagnetized dusty plasma with two species of positive ions. The extremely massive, micron-seized, cold and negatively charged dust particles are described by fluid equations, whereas the two species of positive ions, namely the cold (heavy) and hot (light) ions are described by the kinetic Vlasov equations. Following Ott and Sudan [Phys. Fluids 12, 2388 (1969)], and by considering lower and higher-order perturbations, the evolution of DASWs with Landau damping is shown to be governed by Korteweg-de Vries $(\mathrm{KdV})$, modified $\mathrm{KdV}(\mathrm{mKdV})$ or Gardner (KdV-mKdV)-like equations. The properties of the phase velocity and the Landau damping rate of DASWs are studied for different values of the ratios of the temperatures $(\sigma)$ and the number densities $(\mu)$ of hot and cold ions as well the cold to hot ion mass ratio $m$. The distinctive features of the decay rates of the amplitudes of the $\mathrm{KdV}, \mathrm{mKdV}$ and Gardner solitons with a small effect of Landau damping are also studied in different parameter regimes. It is found that the Gardner soliton points to lower wave amplitudes than the $\mathrm{KdV}$ and $\mathrm{mKdV}$ solitons. The results may be useful for understanding the localization of solitary pulses and associated wave damping (collisionless) in laboratory and space plasmas (e.g., the F-ring of Saturn) in which the number density of free electrons is much smaller than that of ions and the heavy, micron seized dust grains are highly charged.
\end{abstract}

PACS numbers: 52.27.Cm, 52.35.Mw, 52.35.Sb

\section{INTRODUCTION}

Dusty plasmas with an admixture of two species of ions are ubiquitous in both space (e.g., Earth's D and lower E regions, Titan's atmosphere, F-ring of Saturn etc.) [1, 2] and laboratory plasmas [3, 4]. In addition to the main positive ion (light or hot), dusty plasmas also contain a second, micron-sized positive or negative ion (heavy or cold), which characterizes the appearance of an additional wave mode in the plasma [3, 4. There are in fact several motivations for investigating collective plasma oscillations and nonlinear coherent structures like solitons and double layers in dusty plasmas with two groups of ions (See, e.g., Refs. 3 9). In planetary rings, in particular, in the F-ring of Saturn an anomalous situation may arise in which massive dust grains capture almost all the electrons from the background plasma, i.e., the number density of free electrons becomes much smaller than the ions [10]. Such density depletion of electrons (by aerosol particles) have also been observed in the summer polar mesosphere at about $85 \mathrm{~km}$ altitude 11 as well as in the laboratory 12 . On the other hand, plasmas with two species of positive ions have been found to have applications in secondary electron emission guns (e.g., used in bacterial inactivation) in which helium and argon are used as the main gas and an auxiliary gas for ionizing helium atoms 13 .

In dusty plasmas, the size of the dust grains is, in general, not a constant but may vary in the range of $0.05-10 \mu \mathrm{m}$, their mass $m_{d} \sim\left(10^{6}-10^{12}\right) m_{i}$, where

\footnotetext{
* apmisra@visva-bharati.ac.in; apmisra@gmail.com
}

$m_{i}$ is the ion mass, and they have atomic numbers $z_{d} \sim 10^{3}-10^{5}$ (See,e.g., Ref. 14). It is well known that the presence of charged dust grains modifies the dispersion properties of plasma waves and gives rise to a new low-frequency wave mode, called dust-acoustic wave (DAW), which was first theoretically predicted by Rao et al. 15. These waves, associated with the motion of highly charged and massive dust grains, occur at relatively long time $(\sim 0.1 \mathrm{~s})$ and space scales $(\sim 1 \mathrm{~cm})$. Furthermore, in dusty plasmas, the presence of second ion species can significantly modify not only the dispersion properties of DAWs but also some nonlinear coherent structures (See, e.g., Refs. 6 9). These waves can admit collisionless damping due to the resonance interaction of trapped and/or free particles with the wave, i.e., when the particle's velocity becomes closer to the wave phase velocity 16. Such wave damping was first theoretically predicted by Landau 16 in 1946, and later confirmed experimentally by Malmberg et al. [17. Motivated by this invention, Ott and Sudan [18, 19] first studied the effects of (linear) electron Landau damping on the nonlinear propagation of ion-acoustic solitons in electron-ion plasmas. They had neglected the particle's trapping effects on the assumption that the particle trapping time is much longer than that of Landau damping. Their evolution equation was in the form of a Korteweg-de Vries $(\mathrm{KdV})$ equation which contains, apart from the lowest order nonlinear terms and the term that corresponds to linear dispersion relation of ion-acoustic waves, a source term that models the lowest-order effects of resonant particles. In their work, they showed that a wave steepening of an initial wave form may occur or not depending on the relative size of the nonlinearity compared to the Lan- 
dau damping. The latter was also shown to slow down the wave amplitude with time.

Recently, a number of attempts have been made to study the effects of Landau damping on nonlinear solitary waves owing to its importance in different plasma environments. To mention few, Mukherjee et al. [20] studied the effects of linear Landau damping on the nonlinear ion-acoustic solitons in a quantum plasma with the description of $\mathrm{KdV}$ equation. The similar investigation was made by Barman et al. 21] for nonlinear dust-acoustic solitary waves in a dusty pair-ion plasma. In a linear theory, the effects of dust size distribution on the Landau damping rates have also been studied by Zhang et al. in a multi-component dusty plasma 22. However, all the above works were based on either $\mathrm{KdV}$ equation for nonlinear waves or linear dispersion laws for damping rates. As is well known that the $\mathrm{KdV}$ solitons are formed for parameter values well below the critical values (at which the nonlinear coefficient of the KdV equation vanishes) and the modified $\mathrm{KdV}(\mathrm{mKdV})$ solitons are formed for parameter values close to the critical values. However, when both the $\mathrm{KdV}$ and $\mathrm{mKdV}$ equations fail, Gardner (or KdV-mKdV) equation, which generalizes the $\mathrm{KdV}$ and $\mathrm{mKdV}$ equations, is more appropriate to describe the evolution of solitary waves in plasmas. Such (Gardner) solitons are formed for parameters very close to and around the critical values of the plasma parameters. Though, there are a number of works in the literature dealing with the Gardner solitons in plasmas (See, e.g., Ref. 23), however, all are based on fluid theory approach and thus without the effects of Landau damping.

In this work, we investigate the Landau damping effects on dust-acoustic (DA) solitary waves (SWs) (DASWs) in a dusty plasmas with two different species of positive ions namely heavy or cold and light or hot ions. Following Ott and Sudan [18, 19, we derive a KdV as well as $\mathrm{mKdV}$ and Gardner equations (with Landau damping) by considering lower and higher order nonlinearities to describe the evolution of dust-acoustic solitary waves for parameter values far, near and around their critical values. The properties of the $\mathrm{KdV}, \mathrm{mKdV}$ and Gardner solitons with the effects of Landau damping, the linear phase velocity as well as the Landau damping rate are also analyzed with the effects of the ratios of the number densities $(\mu)$ and temperatures $(\sigma)$ of hot to cold ions as well as cold to hot ion mass ratio $m$. It is shown that the landau damping has an effect of slowing down the wave amplitude with time, and the Gardner soliton points to lower wave amplitude.

\section{BASIC EQUATIONS}

We consider the nonlinear propagation of dust-acoustic waves (DAWs) in an unmagnetized collisionless electron free dusty plasma which consists of extremely massive, negatively charged, micron-seized cold dust fluids and singly charged hot (light) and cold (heavy) positive ions. We assume that the dust particles have constant mass and charge. The latter, in general, can vary and introduce a new low-frequency wave eigenmode as well as a dissipative effect (other than the wave damping) into the system. However, we neglect this charge fluctuation effect on the assumption that the charging rate of dust grains is very high compared to the dust plasma oscillation frequency. We also assume that the size of the dust grains is small compared to the average interparticle distance, and the ratio of electric charge to mass of the dust grains remains much smaller than those of ions. At equilibrium, the overall charge neutrality condition satisfies

$$
n_{c 0}+n_{h 0}=z_{d} n_{d 0},
$$

where $n_{j 0}$ is the unperturbed number density of species $j$ (where $j=c, h$ and $d$, respectively, stand for cold ions, hot ions and charged dust grains) and $z_{d}(>0)$ is the unperturbed dust charge state.

The basic equations for the dynamics of charged dust particles together with cold and hot ions in one space dimension are

$$
\begin{gathered}
\partial_{t} n_{d}+\partial_{x}\left(n_{d} u_{d}\right)=0, \\
\partial_{t} u_{d}+u_{d} \partial_{x} u_{d}=-\left(q_{d} / m_{d}\right) \partial_{x} \phi, \\
\partial_{t} f_{j}+v \partial_{x} f_{j}-\left(q_{j} / m_{j}\right) \partial_{x} \phi \partial_{v} f_{j}=0, \\
\partial_{x}^{2} \phi=-4 \pi e\left(n_{c}+n_{h}-z_{d} n_{d}\right),
\end{gathered}
$$

where $\partial_{\alpha} f$ denotes partial differentiation of $f$ with respect to $\alpha$. Also, Eq. (4) is for the cold $(j=c)$ and hot $(j=h)$ ions with their number densities given by

$$
n_{j}=\int_{-\infty}^{\infty} f_{j} d v .
$$

In equations (2)-(6), the symbols $n_{d}, u_{d}, q_{d}\left(=-z_{d} e, e\right.$ being the elementary charge), $m_{d}$, respectively, denote the number density, fluid velocity, charge and mass of dust grains. Also, $v$ is the particle's velocity, $f_{j}$ is the velocity distribution function and $m_{j}$ is the mass of hot and cold ions. Furthermore, $q_{j}=e, \phi$ is the electrostatic potential, and $x$ and $t$ are the space and time coordinates.

In what follows, we normalize the physical quantities according to $u_{d} \rightarrow u_{d} / c_{s}, \phi \rightarrow e \phi / k_{B} T_{h}, n_{j} \rightarrow n_{j} / n_{j 0}$, $n_{d} \rightarrow n_{d} / n_{d 0}, f_{j} \rightarrow f_{j} v_{t j} / n_{j 0}, v \rightarrow v / v_{t h}$, where $c_{s}=\sqrt{z_{d} k_{B} T_{h} / m_{d}}=\omega_{p d} \lambda_{D}$ is the DA speed with $\omega_{p d}=$ $\sqrt{4 \pi n_{d 0} z_{d}^{2} e^{2} / m_{d}}$ and $\lambda_{D}=\sqrt{k_{B} T_{h} / 4 \pi n_{d 0} z_{d} e^{2}}$ denoting, respectively, the dust plasma frequency and the plasma Debye length. Here, $k_{B}$ is the Boltzmann constant, $T_{j}$ is the thermodynamic temperature and $v_{t j}\left(=\sqrt{k_{B} T_{j} / m_{j}}\right)$ is the thermal velocity of $j$-species particles. The space and time variables are normalized by $L$ and $L / c_{s}$ respectively, where $L$ is the characteristic scale length for variations of the dependent variables $n_{j}, u_{d}, \phi, f_{j}$ etc. Thus, 
from Eqs. (2)- $(6)$, we obtain the following set of equations in dimensionless form:

$$
\begin{gathered}
\partial_{t} n_{d}+\partial_{x}\left(n_{d} u_{d}\right)=0 \\
\partial_{t} u_{d}+u_{d} \partial_{x} u_{d}=\partial_{x} \phi \\
\delta \partial_{t} f_{j}+v \partial_{x} f_{j}-\left(m_{h} / m_{j}\right) \partial_{x} \phi \partial_{v} f_{j}=0, \\
\left(\lambda_{D}^{2} / L^{2}\right) \partial_{x}^{2} \phi=-\left(\mu_{c d} n_{c}+\mu h d n_{h}+n_{d}\right), \\
n_{j}=\sqrt{m_{j} T_{h} / m_{h} T_{j}} \int_{-\infty}^{\infty} f_{j} d v,
\end{gathered}
$$

where $\delta=\sqrt{z_{d} m_{h} / m_{d}}$ and $\mu_{j d}=n_{j 0} / z_{d} n_{d 0}$ is the density ratio for $j=c, h$. The charge neutrality condition (1) reduces to

$$
\mu_{c d}+\mu_{h d}=1, \text { or } \mu_{h d}=\mu /(1+\mu),
$$

where $\mu=n_{h 0} / n_{c 0}$ is the ratio of hot to cold unperturbed ion number densities. As in Ref. 21, there are three basic parameters. The first one is the ratio $\delta \equiv \sqrt{z_{d} m_{h} / m_{d}}$ or $m \delta\left(m=m_{c} / m_{h}\right)$, which represents the finite inertial effects of hot and cold ions, and, in particular, the Landau damping. The second one is the ratio of perturbed dust density to its equilibrium value $n_{d 1} / n_{d 0}$, which measures the strength of nonlinearity in electrostatic perturbations. Lastly, the parameter $\lambda_{D} / L$, measuring the strength of the wave dispersion due to deviation from the quasineutrality. This parameter will eventually disappear in the left-hand side of Eq. (10), if one considers the normalization of $x$ by $\lambda_{D}$ instead of $L$. Note that since the dust particles are assumed to be cold, the Landau damping effects are provided solely by the hot and cold ions, and the damping rate is $\propto \delta$.

\section{DERIVATION OF KDV EQUATION}

Before going to derive the KdV equation we first consider the following scaling for the basic parameters described in the previous section as [18, 21]

$$
\delta=\alpha_{1} \epsilon, \quad n_{d 1} / n_{d 0}=\alpha_{2} \epsilon, \quad \lambda_{D}^{2} / L^{2}=\alpha_{3} \epsilon,
$$

where $\epsilon(\lesssim 1)$ is a smallness positive parameter and $\alpha_{j} \sim$ $o(1)(j=1,2,3)$ is a constant. Under the scaling (13) Eq. (9) for hot and cold ions reduces to

$$
\alpha_{1} \epsilon \partial_{t} f_{h}+v \partial_{x} f_{h}-\partial_{x} \phi \partial_{v} f_{h}=0
$$

and

$$
\alpha_{1} \epsilon \partial_{t} f_{c}+v \partial_{x} f_{c}-(1 / m) \partial_{x} \phi \partial_{v} f_{c}=0 .
$$

Next, in the small-amplitude limit (i.e., $\epsilon \rightarrow 0$ ) in which inertial effects of hot and cold ions are neglected, and for $L \gg \lambda_{D}$ (valid for long-wavelength perturbations), Eqs. (7)- 10 yield the following linear dispersion law 21

$$
v_{p} \equiv \omega / k=\left(\mu_{h d}+\sigma \mu_{c d}\right)^{-1 / 2},
$$

where $\omega(k)$ is the wave frequency (number) of the plane wave perturbations and $\sigma=T_{h} / T_{i}$ is the ratio of hot to cold ion temperatures. Equation 16 has the same form as Eq. (15) in Ref. 21 obtained in dusty plasmas with positive and negative ions. From Eq. 16) it is seen that the DAWs become dispersionless in the long-wavelength oscillations with the constant phase speed $v_{p}\left(<c_{s}\right)$. This implies that the time derivatives of all physical quantities should vanish in a frame moving at the speed $v_{p}$, and one can thus expect slow variations of the wave amplitude in it. So, we introduce the stretched coordinates as 24]

$$
\xi=\epsilon^{1 / 2}(x-M t), \tau=\epsilon^{3 / 2} t,
$$

where $M$ is the nonlinear wave speed (relative to the frame with $x$ and $t$ as coordinates) normalized by $c_{s}$, to be shown to be equal to $v_{p}$ later. The dynamical variables are expanded as

$$
\begin{aligned}
n_{d} & =1+\alpha_{2} \epsilon n_{d}^{(1)}+\alpha_{2}^{2} \epsilon^{2} n_{d}^{(2)}+\cdots, \\
u_{d} & =\alpha_{2} \epsilon u_{d}^{(1)}+\alpha_{2}^{2} \epsilon^{2} u_{d}^{(2)}+\cdots, \\
\phi & =\alpha_{2} \epsilon \phi^{(1)}+\alpha_{2}^{2} \epsilon^{2} \phi^{(2)}+\cdots, \\
n_{j} & =1+\alpha_{2} \epsilon n_{j}^{(1)}+\alpha_{2}^{2} \epsilon^{2} n_{j}^{(2)}+\cdots, \\
f_{j} & =f_{j}^{(0)}+\alpha_{2} \epsilon f_{j}^{(1)}+\alpha_{2}^{2} \epsilon^{2} f_{j}^{(2)}+\cdots,
\end{aligned}
$$

where the equilibrium distribution function $f_{j}^{(0)}, j=$ $c, h$, are assumed to be the Maxwellian

$$
f_{j}^{(0)}=\sqrt{1 / 2 \pi} \exp \left[\left(m_{j} T_{e} / m_{e} T_{j}\right)\left(-v^{2} / 2\right)\right] .
$$

In the next step we substitute the expressions from Eqs. (17) and (18) into Eqs. (7), (8), (10), (11), (14) and (15), and equate different powers of $\epsilon$. In this way, we obtain the equations for first and second order perturbations. Since the method and intermediate steps for the derivation of the $\mathrm{KdV}$ equation are almost similar to that in Ref. 21, we skip presenting first and second order expressions for the perturbations. However, those can be given for the derivation of the Gardner equation. The mKdV equation will then directly follow from the Gardner equation. Thus, following Ref. 21, we obtain the following $\mathrm{KdV}$ equation

$$
\frac{\partial n}{\partial \tau}+a \mathrm{P} \int_{-\infty}^{\infty} \frac{\partial n}{\partial \xi^{\prime}} \frac{d \xi^{\prime}}{\xi-\xi^{\prime}}+b n \frac{\partial n}{\partial \xi}+c \frac{\partial^{3} n}{\partial \xi^{3}}=0,
$$

where the coefficients of the Landau damping $(a)$, the nonlinear $(b)$ and the dispersive term $(c)$, respectively, are given by

$$
a=\left(\alpha_{1} / \sqrt{8 \pi \sigma} \sigma_{1}^{2}\right)\left[\sqrt{m}-\left(\sqrt{m}-\sigma^{-3 / 2}\right) \mu_{h d}\right],
$$




$$
\begin{gathered}
b=\left(\alpha_{2} / 2 \sqrt{\sigma \sigma_{1}}\right)\left(3-\sigma_{2} / \sigma_{1}^{2}\right) . \\
c=\left(\alpha_{3} / 2\right)\left(\sigma \sigma_{1}\right)^{-3 / 2},
\end{gathered}
$$

with $\sigma_{1}=1+\left(\sigma^{-1}-1\right) \mu_{h d} \equiv \mu_{c d}(\sigma+\mu) / \sigma, \sigma_{2}=$ $1+\left(\sigma^{-2}-1\right) \mu_{h d} \equiv \mu_{c d}\left(\sigma^{2}+\mu\right) / \sigma^{2}$ and $\sigma_{3}=1+\left(\sigma^{-3}-\right.$ 1) $\mu_{h d} \equiv \mu_{c d}\left(\sigma^{3}+\mu\right) / \sigma^{3}$. We note that the nonlinear coefficient $b$ of the $\mathrm{KdV}$ equation (20) vanishes for $R_{1} \equiv 3-\sigma_{2} / \sigma_{1}^{2} \equiv 3-(1+\mu)\left(\sigma^{2}+\mu\right) /(\sigma+\mu)^{2}=0$ along a curve in the $\sigma-\mu$ plane (See Fig. 11). In this case, the $\mathrm{KdV}$ equation fails to describe the nonlinear evolution of DASWs, and one has to look for some higher order perturbations, i.e., the evolution of DASWs can then be described by $\mathrm{mKdV}$ or Gardner equations to be obtained shortly. Furthermore, in absence of the Landau damping

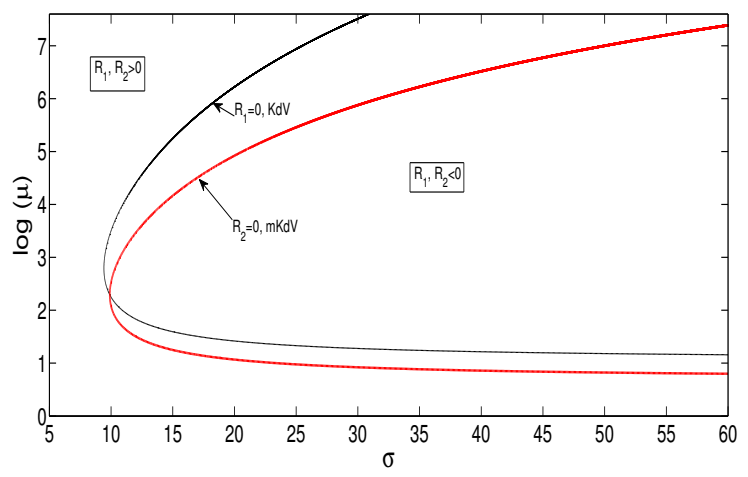

FIG. 1. Contour plots of $R_{1} \equiv 3-\sigma_{2} / \sigma_{1}^{2} \equiv 3-(1+$ $\mu)\left(\sigma^{2}+\mu\right) /(\sigma+\mu)^{2}=0$ (The thin or black curve) [i.e., vanishing of the nonlinear coefficients $b$ and $b_{1}$ of the KdV and Gardner equations (20) and [87] and $R_{2} \equiv 1-\sigma_{3} / 15 \sigma_{1}^{3} \equiv$ $1-(1+\mu)^{2}\left(\sigma^{3}+\mu\right) /(\sigma+\mu)^{3}=0$ (The thick or red curve) [i.e., vanishing of the nonlinear coefficient $b_{2}$ of the $\mathrm{mKdV}$ equation [90] showing the critical regions in the $\sigma-\mu$ plane. There is a common critical point $(\sigma, \mu) \approx(10,10)$ at which both the $\mathrm{KdV}$ and $\mathrm{mKdV}$ equations fail to describe the evolution of DAWs.

the KdV equation admits compressive or rarefactive soliton solutions according to when $b>0(b<0)$, i.e., when $b$ falls in the region $R_{1}>0\left(R_{1}<0\right)$. We will, however, later see that the region for which $b<0$ is inadmissible for the existence of $\mathrm{KdV}$ solitons with a small effect of the Landau damping [See Eq. (120) in Sec. VI]. We also mention that the KdV solitons with Landau damping are formed for values of the parameters $\sigma$ and $\mu$ far below the critical values along the curve $R_{1}=0$, the $\mathrm{mKdv}$ solitons are formed for the parameters close to the critical values and Gardner solitons are formed approximately at and around the critical values of $\sigma$ and $\mu$. Thus, the $\mathrm{KdV}$ equation may be valid for the parameters $\sigma$ and $\mu$ satisfying $R_{1} \sim o(1)$. However, in the case of $R_{1} \sim o(\epsilon)$ or $R_{1} \approx 0$, one has to deal with $\mathrm{mKdV}$ or Gardner equations which involve higher-order (of $\epsilon$ ) perturbations for the evolution of dust-acoustic solitary waves in the plasma.

Figure 1 shows the contour plot of $R_{1}=0$ (the thin or black curve) in the $\sigma-\mu$ plane in which the nonlinear co- efficients $b$ and $b_{1}$, respectively, of the KdV and Gardner (obtained later) equations (20) and (87) vanish. The plot of $R_{2}=0$ (The thick or red curve) represents the same, however, for the vanishing of the nonlinear coefficient $b_{2}$ of the $\mathrm{mKdV}$ equation (90) (shown later). We find that when $\sigma$ and $\mu$ assume values satisfying $R_{1} \approx 0$ the $\mathrm{KdV}$ solitons may not exist, the evolution of dust-acoustic solitons can then be described by the $\mathrm{mKdV}$ equation. The latter can also be inappropriate for the evolution of solitons when $R_{2} \approx 0$ for some values of $\sigma$ and $\mu$. From Fig. 1 it is also seen that the curves for $R_{1}=0$ and $R_{2}=0$ has a common point of intersection $(\sigma, \mu) \approx(10,10)$ at which both the $\mathrm{KdV}$ and $\mathrm{mKdV}$ solitons cease to exist. Also, the lower parts of these curves below the intersection point are close to each other, implying that the parameters close to the lower part of $R_{1}=0$ may be some what close to that of $R_{2}=0$. In this case, the Gardner equation is necessary for the evolution of DASWs, which will be shown later.

\section{DERIVATION OF GARDNER AND MKDV EQUATIONS}

To derive the Gardner equation for DASWs we consider $R_{1} \sim o(\epsilon)$. In this case, the scaling for the basic parameters [Eq. [13] ] and the equations for hot and cold ions, i.e., Eqs. (14) and (15) are to be changed by the substitution $\epsilon \rightarrow \epsilon^{2}$ as we are looking for perturbations with higher-order (of $\epsilon$ ) effects. Thus, we have the modified scaling for the basic parameters

$$
\delta=\alpha_{1} \epsilon^{2}, \quad n_{d 1} / n_{d 0}=\alpha_{2} \epsilon^{2}, \quad \lambda_{D}^{2} / L^{2}=\alpha_{3} \epsilon^{2} .
$$

The modified kinetic equations for ions are

$$
\alpha_{1} \epsilon^{2} \partial_{t} f_{h}+v \partial_{x} f_{h}-\partial_{x} \phi \partial_{v} f_{h}=0,
$$

and

$$
\alpha_{1} \epsilon^{2} \partial_{t} f_{c}+v \partial_{x} f_{c}-(1 / m) \partial_{x} \phi \partial_{v} f_{c}=0 .
$$

Also, the stretched coordinates are redefined as

$$
\xi=\epsilon(x-M t), \tau=\epsilon^{3} t,
$$

However, the dynamical variables are expanded in the same way as Eq. 18 for the KdV equation. Next, we substitute the expressions from Eqs. (18) and (27) into Eqs. (7), (8), (10), (11), (25) and (26) and equate different powers of $\epsilon$. We temporarily drop the constant $\alpha_{2}$ in the subsequent expressions and equations. However, we note that the constants $\alpha_{1}, \alpha_{2}$ and $\alpha_{3}$ will explicitly appear in the coefficients of the Landau damping, nonlinear and the dispersive terms in the evolution equation. The results will be given in the following subsections.

\section{A. First-order perturbations and nonlinear wave speed}

We successively equate the coefficients of $\epsilon^{2}$ from Eqs. (7) and (8), the coefficients of $\epsilon$ from Eqs. (10) and (11), 
and the coefficients of $\epsilon^{2}$ from Eqs. 25 and 26 to obtain

$$
\begin{gathered}
n_{d}^{(1)}=u_{d}^{(1)} / M \\
u_{d}^{(1)}=-\phi^{(1)} / M \\
0=-\left(\mu_{c d} n_{c}^{(1)}+\mu_{h d} n_{h}^{(1)}\right)+n_{d}^{(1)}, \\
n_{j}^{(1)}=\sqrt{m_{j} T_{h} / m_{h} T_{j}} \int_{-\infty}^{\infty} f_{j}^{(1)} d v, \\
v \partial_{\xi} f_{h}^{(1)}+v f_{h}^{(0)} \partial_{\xi} \phi^{(1)}=0, \\
v \partial_{\xi} f_{c}^{(1)}+\sigma v f_{c}^{(0)} \partial_{\xi} \phi^{(1)}=0 .
\end{gathered}
$$

Equations (28) and 29 reduce to

$$
n_{d}^{(1)}=-\phi^{(1)} / M^{2} \text {. }
$$

Also, Eq. 32 yields the following relation [18]

$$
\partial_{\xi} f_{h}^{(1)}=-f_{h}^{(0)} \partial_{\xi} \phi^{(1)}+\lambda(\xi, \tau) \delta(v),
$$

where $\delta(v)$ is the Dirac delta function and $\lambda(\xi, \tau)$ is an arbitrary function of $\xi$ and $\tau$ implying that the solution for $\partial f_{h}^{(1)} / \partial \xi$ is not unique. Thus, for the unique solution to exist we add a higher-order term $\epsilon^{6} \alpha_{1}\left(\partial f_{h}^{(1)} / \partial \tau\right)$ originating from the term $\epsilon^{5} \alpha_{1}\left(\partial f_{h} / \partial \tau\right)$ in Eq. 25) after the expressions (27) and (18) being substituted. Thus, we rewrite Eq. (32) as 18

$$
\alpha_{1} \epsilon^{2} \partial_{\tau} f_{h \epsilon}^{(1)}+v \partial_{\xi} f_{h \epsilon}^{(1)}=-v f_{h}^{(0)} \partial_{\xi} \phi^{(1)},
$$

Similarly, from Eq. (33), we obtain

$$
\alpha_{1} \epsilon^{2} \partial_{\tau} f_{c \epsilon}^{(1)}+v \partial_{\xi} f_{c \epsilon}^{(1)}=-\sigma v f_{c}^{(0)} \partial_{\xi} \phi^{(1)} .
$$

The unique solutions of Eqs. (36) and (37) can be given as

$$
f_{j}^{(1)}=\lim _{\epsilon \rightarrow 0} f_{j \epsilon}^{(1)}
$$

where $f_{j \epsilon}^{(1)}(j=h, c)$ are to be obtained by taking the Fourier transform of Eq. (36) with respect to $\xi$ and $\tau$ according to the formula

$$
\hat{f}(\omega, k)=\int_{-\infty}^{\infty} \int_{-\infty}^{\infty} f(\xi, \tau) e^{i(k \xi-\omega \tau)} d \xi d \tau
$$

Thus, we obtain

$$
\hat{f}_{h \epsilon}^{(1)}=-k v f_{h}^{(0)}\left(k v-\epsilon^{2} \alpha_{1} \omega\right)^{-1} \hat{\phi}^{(1)} .
$$

Next, to remove the singularity in Eq. 40 we replace $\omega$ by $\omega+i \eta$ with $\eta(>0)$ being small, and obtain

$$
\hat{f}_{h \epsilon}^{(1)}=-k v f_{h}^{(0)}\left[\left(k v-\epsilon^{2} \alpha_{1} \omega\right)-i \eta \alpha_{1} \epsilon^{2}\right]^{-1} \hat{\phi}^{(1)} .
$$

Proceeding to the limit as $\epsilon \rightarrow 0$, using the Plemelj's formula (in which $P$ denotes the Cauchy principal value)

$$
\lim _{\epsilon \rightarrow 0}(x+i \epsilon)^{-1}=-i \pi \delta(x)+\mathrm{P}(1 / x),
$$

and noting that $x \mathrm{P}(1 / x)=1, x \delta(x)=0$, we obtain

$$
\hat{f}_{h}^{(1)}=-f_{h}^{(0)} \hat{\phi}^{(1)} .
$$

Next, we take the Fourier inversion of Eq. (43) to obtain

$$
f_{h}^{(1)}=-f_{h}^{(0)} \phi^{(1)}
$$

Similar expression for cold ions can also be obtained from Eq. (37) as

$$
f_{c}^{(1)}=-\sigma f_{c}^{(0)} \phi^{(1)} .
$$

Substituting the expressions from Eqs. (44) and 45 into Eq. (31), we obtain

$$
\begin{gathered}
n_{h}^{(1)}=-\phi^{(1)}, \\
n_{c}^{(1)}=-\sigma \phi^{(1)} .
\end{gathered}
$$

The expressions for $n_{j}^{(1)}(j=c, h, d)$ from Eqs. 34, 46 and 47) can now be used in Eq. 30, to obtain the following expression for the nonlinear wave speed 21.

$$
M=\left(\mu_{h d}+\sigma \mu_{c d}\right)^{-1 / 2} \equiv \sqrt{(1+\mu) /(\mu+\sigma)}
$$

Evidently, $M$ has exactly the same expression as $v_{p}$ obtained in the linear dispersion law 16 for plane wave perturbations. Figure 2 shows the characteristics of $M$ with $\sigma$ for different values of the density ratio $\mu$. We note that since $\sigma \equiv T_{h} / T_{c}>1$, the quantity under the square root in Eq. 48 is always smaller than unity, i.e., the DAWs propagate with a phase speed being smaller than the DA speed $c_{s}$. We also find that as the value of $\mu$ increases, the value of the phase velocity $M$ increases. However, the same decreases for increasing values of the temperature ratio $\sigma$, and approaches to zero for $\sigma \gg 1$. The latter is, however, inadmissible in the present theory as it would not give any physical result. We mention that the results in this subsection (up to the linear theory) are the same as for the $\mathrm{KdV}$ and Gardner or mKdV equations. However, the results (nonlinear) will be different in the expressions for second and higher order perturbations.

\section{B. Second-order perturbations}

We successively equate the coefficients of $\epsilon^{3}$ from Eqs. (7) and (8), the coefficients of $\epsilon^{2}$ from Eqs. (10) and (11) as well as the coefficients of $\epsilon^{3}$ from Eqs. (14) and (15) to obtain

$$
-M n_{d}^{(2)}+u_{d}^{(2)}=-n_{d}^{(1)} u_{d}^{(1)}
$$




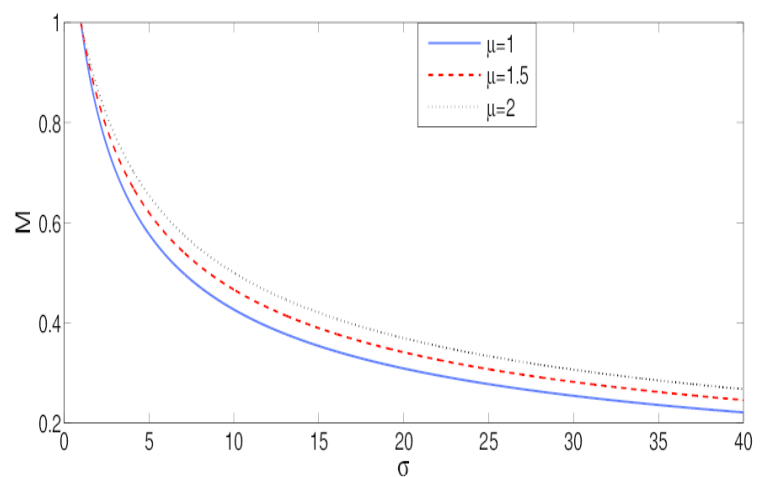

FIG. 2. The linear phase velocity $M$ [Eq. (48)] of DAWs is shown with respect to the temperature ratio $\sigma\left(=T_{h} / T_{c}\right)$ for different values of the density ratio $\mu\left(=n_{h 0} / n_{c 0}\right)$ as in the figure.

$$
\begin{gathered}
u_{d}^{(2)}=-(1 / M) \phi^{(2)}+\left(1 / 2 M^{3}\right)\left(\phi^{(1)}\right)^{2}, \\
n_{d}^{(2)}=\mu_{c d} n_{c}^{(2)}+\mu_{h d} n_{h}^{(2)} \\
n_{j}^{(2)}=\sqrt{m_{j} T_{h} / m_{h} T_{j}} \int_{-\infty}^{\infty} f_{j}^{(2)} d v \\
v \partial_{\xi} f_{h}^{(2)}-\partial_{\xi} \phi^{(1)} \partial_{v} f_{h}^{(1)}+v f_{h}^{(0)} \partial_{\xi} \phi^{(2)}=0 \\
v \partial_{\xi} f_{c}^{(2)}-(1 / m) \partial_{\xi} \phi^{(1)} \partial_{v} f_{c}^{(1)}+\sigma v f_{c}^{(0)} \partial_{\xi} \phi^{(2)}=0 .
\end{gathered}
$$

Eliminating $u_{d}^{(2)}$ from Eqs. (49) and (50) and using the first-order expressions from Eqs. 29) and (34), we obtain

$$
n_{d}^{(2)}=\left(3 / 2 M^{4}\right)\left(\phi^{(1)}\right)^{2}-\left(1 / M^{2}\right) \phi^{(2)} .
$$

Substituting the expressions for $f_{j}$ from Eqs. (44) and (45) into Eqs. (53) and (54) we successively obtain

$$
\begin{gathered}
v \partial_{\xi} f_{h}^{(2)}-v f_{h}^{(0)} \phi^{(1)} \partial_{\xi} \phi^{(1)}+v f_{h}^{(0)} \partial_{\xi} \phi^{(2)}=0, \\
v \partial_{\xi} f_{c}^{(2)}-\sigma^{2} v f_{c}^{(0)} \phi^{(1)} \partial_{\xi} \phi^{(1)}+\sigma v f_{c}^{(0)} \partial_{\xi} \phi^{(2)}=0 .
\end{gathered}
$$

As in the previous section, to obtain the unique solutions for $f_{j}^{(2)}$ we introduce a higher-order term $\epsilon^{7} \alpha_{1}\left(\partial f_{j}^{(1)} / \partial \tau\right)$ originating from the term $\epsilon^{5} \alpha_{1}\left(\partial f_{j} / \partial \tau\right)$ in Eqs. 25) and (26) after the expressions (27) and (18) being substituted, and replace $f_{j}^{(2)}$ by $f_{j \epsilon}^{(2)}$. Thus, we rewrite Eqs. (56) and (57) as

$$
\begin{aligned}
& \alpha_{1} \epsilon^{2} \partial_{\tau} f_{h \epsilon}^{(2)}+v \partial_{\xi} f_{h \epsilon}^{(2)}+v f_{h}^{(0)} \partial_{\xi} \phi^{(2)} \\
& =(1 / 2) v f_{h}^{(0)} \partial_{\xi}\left(\phi^{(1)}\right)^{2},
\end{aligned}
$$

$$
\begin{aligned}
& \alpha_{1} \epsilon^{2} \partial_{\tau} f_{c \epsilon}^{(2)}+v \partial_{\xi} f_{c \epsilon}^{(2)}+\sigma v f_{c}^{(0)} \partial_{\xi} \phi^{(2)} \\
& =\left(\sigma^{2} / 2\right) v f_{c}^{(0)} \partial_{\xi}\left(\phi^{(1)}\right)^{2} .
\end{aligned}
$$

As before, the solutions $f_{j}^{(2)}, j=h, c$ can be found uniquely from Eqs. (58) and $(59)$ by the following relation

$$
f_{j}^{(2)}=\lim _{\epsilon \rightarrow 0} f_{j \epsilon}^{(2)} .
$$

So, taking the Fourier transform of Eq. (58) according to the formula (39), and replacing $\omega$ by $\omega+i \eta(\eta>0)$, we obtain

$$
\hat{f}_{h \epsilon}^{(2)}=\left[-\hat{\phi}^{(2)}+\frac{1}{2}\left(\hat{\phi}^{(1)}\right)^{2}\right]\left[\frac{k v f_{h}^{(0)}}{\left(k v-\epsilon^{2} \alpha_{1} \omega\right)-i \eta \alpha_{1} \epsilon^{2}}\right] .
$$

Proceeding to the limit as $\epsilon \rightarrow 0$ and using the Plemelj's formula 42 together with $x \mathrm{P}(1 / x)=1$ and $x \delta(x)=0$, we obtain from Eq. 61) the following

$$
\hat{f}_{h}^{(2)}=\left[-\hat{\phi}^{(2)}+(1 / 2)\left(\hat{\phi}^{(1)}\right)^{2}\right] f_{h}^{(0)} .
$$

Next, the Fourier inverse transform of Eq. 62 yields the following solution for the hot ion distribution function

$$
f_{h}^{(2)}=\left[-\phi^{(2)}+(1 / 2)\left(\phi^{(1)}\right)^{2}\right] f_{h}^{(0)} .
$$

By the same way as above, one can also obtain the similar expression for the cold ion distribution as

$$
f_{c}^{(2)}=\left[-\sigma \phi^{(2)}+\left(\sigma^{2} / 2\right)\left(\phi^{(1)}\right)^{2}\right] f_{c}^{(0)} .
$$

The expressions for the second order perturbations of the number densities can now be obtained from Eq. (52) by using Eqs. 63) and (64) as

$$
n_{h}^{(2)}=-\phi^{(2)}+(1 / 2)\left(\phi^{(1)}\right)^{2},
$$

and

$$
n_{c}^{(2)}=-\sigma \phi^{(2)}+\left(\sigma^{2} / 2\right)\left(\phi^{(1)}\right)^{2} .
$$

Furthermore, substituting the expressions for $n_{d}^{(2)}, n_{h}^{(2)}$, $n_{c}^{(2)}$ from Eqs. (55), (65) and (66) into Eq. (51), we obtain for nonzero perturbations the following relation

$$
\begin{aligned}
& -(1 / 2)\left(\mu_{h d}+\mu_{c d} \sigma^{2}\right)\left(\phi^{(1)}\right)^{2}+\left(\mu_{h d}+\mu_{c d} \sigma\right) \phi^{(2)} \\
& =\left(1 / M^{2}\right) \phi^{(2)}-\left(3 / 2 M^{4}\right)\left(\phi^{(1)}\right)^{2},
\end{aligned}
$$

in which the coefficient of $\phi^{(2)}$ vanishes by the dispersion relation 48 yielding

$$
\widetilde{b} \equiv(1 / 2)\left(\sigma \sigma_{1}\right)^{2}\left(3-\sigma_{2} / \sigma_{1}^{2}\right)\left(\phi^{(1)}\right)^{2}=0,
$$

where $\sigma_{1}=(\sigma+\mu) / \sigma(1+\mu)$ and $\sigma_{2}=\left(\sigma^{2}+\mu\right) / \sigma^{2}(1+\mu)$. Here, we note that since $\phi^{(1)}$ is of the order of $\epsilon$, the factor $R_{1} \equiv 3-\sigma_{2} / \sigma_{1}^{2}$ must be at least of the order of $\epsilon$, which we have already assumed in the beginning of this section. So, the term $\widetilde{b}$ will contribute to the terms in the coefficient of $\epsilon^{3}$ of the Poisson's equation in Sec. IVC [See Eq. (71)], and finally in the coefficient $b_{1}$ of the Gardner equation (87). 


\section{Third-order perturbations}

Equating the coefficients of $\epsilon^{4}$ from Eqs. (7) and (8), the coefficients of $\epsilon^{3}$ from Eqs. 100 and (11), and the coefficients of $\epsilon^{4}$ from Eqs. (25) and (26), we successively obtain

$$
\begin{gathered}
-M \partial_{\xi} n_{d}^{(3)}+\partial_{\tau} n_{d}^{(1)}+\partial_{\xi} u_{d}^{(3)}+\partial_{\xi}\left(n_{d}^{(1)} u_{d}^{(2)}\right) \\
+\partial_{\xi}\left(n_{d}^{(2)} u_{d}^{(1)}\right)=0, \\
\partial_{\tau} u_{d}^{(1)}-M \partial_{\xi} u_{d}^{(3)}+\partial_{\xi}\left(u_{d}^{(1)} u_{d}^{(2)}\right)=\partial_{\xi} \phi^{(3)} . \\
\partial_{\xi}^{2} \phi^{(1)}=-\left(\mu_{h d} n_{h}^{(3)}+\mu_{c d} n_{c}^{(3)}\right)+n_{d}^{(3)} \\
+(1 / 2)\left(\sigma \sigma_{1}\right)^{2}\left(3-\sigma_{2} / \sigma_{1}^{2}\right)\left(\phi^{(1)}\right)^{2}, \\
n_{j}^{(3)}=\sqrt{m_{j} T_{h} / m_{h} T_{j}} \int_{-\infty}^{\infty} f_{j}^{(3)} d v . \\
-\alpha_{1} M \partial_{\xi} f_{h}^{(1)}+v \partial_{\xi} f_{h}^{(3)}+v f_{h}^{(0)} \partial_{\xi} \phi^{(3)} \\
-\partial_{\xi} \phi^{(2)} \partial_{v} f_{h}^{(1)}-\partial_{\xi} \phi^{(1)} \partial_{v} f_{h}^{(2)}=0, \\
-\alpha_{1} M \partial_{\xi} f_{c}^{(1)}+v \partial_{\xi} f_{c}^{(3)}+\sigma v f_{c}^{(0)} \partial_{\xi} \phi^{(3)} \\
-(1 / m)\left(\partial_{\xi} \phi^{(2)} \partial_{v} f_{c}^{(1)}+\partial_{\xi} \phi^{(1)} \partial_{v} f_{c}^{(2)}\right)=0 .
\end{gathered}
$$

As before, to get the unique solutions for $f_{j}^{(3)}$ (for $j=$ $h, c)$, we introduce a higher-order term $\epsilon^{8} \alpha_{1}\left(\partial f_{j}^{(3)} / \partial \tau\right)$ originating from the term $\epsilon^{5} \alpha_{1}\left(\partial f_{j} / \partial \tau\right)$ in Eqs. (25) and (26), and then replacing $f_{j}^{(3)}$ by $f_{j \epsilon}^{(3)}$, where

$$
f_{j}^{(3)}=\lim _{\epsilon \rightarrow 0} f_{j \epsilon}^{(3)},
$$

we obtain from Eqs. 73 and 74 as

$$
\begin{aligned}
& \alpha_{1} \epsilon^{2} \partial_{\tau} f_{h \epsilon}^{(3)}+v \partial_{\xi} f_{h \epsilon}^{(3)}+v f_{h}^{(0)} \partial_{\xi} \phi^{(3)} \\
& =\left(D_{h a}+v D_{h b}\right) f_{h}^{(0)}, \\
& \alpha_{1} \epsilon^{2} \partial_{\tau} f_{c \epsilon}^{(3)}+v \partial_{\xi} f_{c \epsilon}^{(3)}+\sigma v f_{c}^{(0)} \partial_{\xi} \phi^{(3)} \\
& =\left(D_{c a}+v D_{c b}\right) f_{c}^{(0)},
\end{aligned}
$$

where we have used Eqs. (44) and (63) to obtain Eq. (76), and Eqs. (45) and (64) to obtain Eq. (77). The expressions for $D_{h a}, D_{h b}, D_{c a}$ and $D_{c b}$ are given by

$$
\begin{aligned}
& D_{h a}=-\alpha_{1} M \partial_{\xi} \phi^{(1)}, \\
& D_{h b}=-(1 / 6) \partial_{\xi}\left(\phi^{(1)}\right)^{3}+\partial_{\xi}\left(\phi^{(1)} \phi^{(2)}\right), \\
& D_{c a}=-\alpha_{1} M \sigma \partial_{\xi} \phi^{(1)}, \\
& D_{c b}=-\left(\sigma^{3} / 6\right) \partial_{\xi}\left(\phi^{(1)}\right)^{3}+\sigma^{2} \partial_{\xi}\left(\phi^{(1)} \phi^{(2)}\right) .
\end{aligned}
$$

Next, taking the Fourier transforms of Eq. 76 with respect to $\xi$ and $\tau$ according to the formula $(39)$ and replacing $\omega$ by $\omega+i \eta(\eta>0)$, we obtain

$$
\hat{f}_{h \epsilon}^{(3)}=-\left[\frac{k v f_{h}^{(0)} \hat{\phi}^{(3)}+i\left(\hat{D}_{h a}+v \hat{D}_{h b}\right) f_{h}^{(0)}}{\left(k v-\epsilon^{2} \alpha_{1} \omega\right)-i \eta \alpha_{1} \epsilon^{2}}\right] .
$$

In the limit of $\epsilon \rightarrow 0$, Eq. 79 yields

$$
\begin{aligned}
& \hat{f}_{h}^{(3)}+f_{h}^{(0)} \hat{\phi}^{(3)}=-i[\mathrm{P}(1 / k v)+i \pi(\operatorname{sgn} k / k) \delta(v)] \times \\
& \left(\hat{D}_{h a}+v \hat{D}_{h b}\right) f_{h}^{(0)},
\end{aligned}
$$

where we have used the Plemelj's formula 42 together with $x \mathrm{P}(1 / x)=1, x \delta(x)=0$ and $\delta(k v)=(\operatorname{sgn} k / k) \delta(v)$. Now, multiplying both sides of Eq. 80 by $i k$ and then integrating with respect to $v$, we obtain

$$
i k\left(\hat{n}_{h}^{(3)}+\hat{\phi}^{(3)}\right)=\hat{D}_{h b}+i \sqrt{\pi / 2} \operatorname{sgn}(k) \hat{D}_{h a},
$$

which under the Fourier inverse transform reduces to

$$
\begin{aligned}
\partial_{\xi} n_{h}^{(3)}+\partial_{\xi} \phi^{(3)}= & \partial_{\xi}\left(\phi^{(1)} \phi^{(2)}\right)-(1 / 6) \partial_{\xi}\left(\phi^{(1)}\right)^{3} \\
& +\sqrt{\pi / 2} F^{-1}\left(i \operatorname{sgn}(k) \hat{D}_{h a}\right) .
\end{aligned}
$$

Here, $F^{-1}$ stands for the Fourier inverse transform. Using the convolution theorem of Fourier transform and noting that $F^{-1}[i \operatorname{sgn}(k)]=-(1 / \pi) \mathrm{P}(1 / \xi)$, we obtain from Eq. 82

$$
\begin{aligned}
\partial_{\xi} n_{h}^{(3)} & +\partial_{\xi} \phi^{(3)}=\partial_{\xi}\left(\phi^{(1)} \phi^{(2)}\right)-(1 / 6) \partial_{\xi}\left(\phi^{(1)}\right)^{3} \\
& +\alpha_{1} M(1 / \sqrt{2 \pi}) P \int_{-\infty}^{\infty} \partial_{\xi^{\prime}} \phi^{(1)} \frac{d \xi^{\prime}}{\xi-\xi^{\prime}} .
\end{aligned}
$$

Similar equation for the third-order perturbations can be obtained for cold ions as

$$
\begin{aligned}
& (1 / \sqrt{m \sigma}) \partial_{\xi} n_{c}^{(3)}+\sqrt{\frac{\sigma}{m}} \partial_{\xi} \phi^{(3)}=\frac{1}{\sqrt{m \sigma}}\left[\sigma^{2} \partial_{\xi}\left(\phi^{(1)} \phi^{(2)}\right)\right. \\
& \left.-\frac{\sigma^{3}}{6} \partial_{\xi}\left(\phi^{(1)}\right)^{3}\right]+\frac{\alpha_{1} M \sigma}{\sqrt{2 \pi}} P \int_{-\infty}^{\infty} \partial_{\xi^{\prime}} \phi^{(1)} \frac{d \xi^{\prime}}{\xi-\xi^{\prime}} .
\end{aligned}
$$

\section{Gardner and mKdV equations with Landau damping}

In order to obtain the evolution (Gardner) equation for first-order perturbations, we first eliminate $u_{d}^{(3)}$ and $n_{d}^{(3)}$ from Eqs. 69- 71 to obtain

$$
\begin{aligned}
& \partial_{\xi}^{3} \phi^{(1)}=-\mu_{h d} \partial_{\xi} n_{h}^{(3)}-\mu_{c d} \partial_{\xi} n_{c}^{(3)}-\left(1 / M^{2}\right) \partial_{\xi} \phi^{(3)} \\
& +(1 / M)\left[\partial_{\tau} n_{d}^{(1)}+(1 / M)\left(\partial_{\tau} u_{d}^{(1)}+\partial_{\xi}\left(u_{d}^{(1)} u_{d}^{(2)}\right)\right)\right. \\
& \left.+\partial_{\xi}\left(n_{d}^{(1)} u_{d}^{(2)}+n_{d}^{(2)} u_{d}^{(1)}\right)\right] \\
& +\left(\sigma \sigma_{1}\right)^{2}\left(3-\sigma_{2} / \sigma_{1}^{2}\right) \phi^{(1)} \partial_{\xi} \phi^{(1)}
\end{aligned}
$$


Next, we use Eqs. 83 and 84 to eliminate $n_{h}^{(3)}$ and $n_{c}^{(3)}$ from Eq. 85, and obtain

$$
\begin{aligned}
& \partial_{\xi}^{3} \phi^{(1)}=-\left(\mu_{h d}+\sigma^{2} \mu_{c d}\right) \partial_{\xi}\left(\phi^{(1)} \phi^{(2)}\right) \\
& +(1 / 6)\left(\mu_{h d}+\sigma^{3} \mu_{c d}\right) \partial_{\xi}\left(\phi^{(1)}\right)^{3} \\
& -\frac{\alpha_{1} M}{\sqrt{2 \pi}}\left(\mu_{h d}+\sqrt{m} \sigma^{3 / 2} \mu_{c d}\right) P \int_{-\infty}^{\infty} \partial_{\xi^{\prime}} \phi^{(1)} \frac{d \xi^{\prime}}{\xi-\xi^{\prime}} \\
& +(1 / M)\left[\partial_{\tau} n_{d}^{(1)}+(1 / M)\left(\partial_{\tau} u_{d}^{(1)}+\partial_{\xi}\left(u_{d}^{(1)} u_{d}^{(2)}\right)\right)\right. \\
& \left.+\partial_{\xi}\left(n_{d}^{(1)} u_{d}^{(2)}+n_{d}^{(2)} u_{d}^{(1)}\right)\right] \\
& +\left(\sigma \sigma_{1}\right)^{2}\left(3-\sigma_{2} / \sigma_{1}^{2}\right) \phi^{(1)} \partial_{\xi} \phi^{(1)} .
\end{aligned}
$$

Next, we substitute the expressions of $u_{d}^{(2)}$ and $n_{d}^{(2)}$ from Eqs. (50) and (55) into Eq. (86). Here, we note that the coefficient of $\phi^{(2)} \phi^{(1)}$ is $\sim\left(3-\sigma_{2} / \sigma_{1}^{2}\right) \sim \epsilon$, and so the term $\propto\left(3-\sigma_{2} / \sigma_{1}^{2}\right)\left(\phi^{(2)} \phi^{(1)}\right) \sim \epsilon^{4}$ [since $\phi^{(2)} \sim \epsilon^{2}$ and $\left.\phi^{(1)} \sim \epsilon\right]$. Thus, this term will not contribute to Eq. 86 and we discard it. Finally, we replace $\phi^{(1)}$ by $n \equiv n_{d}^{(1)}$ using Eqs. $(28)$ and $(29)$ to obtain from Eq. (86. the following Gardner (KdV-mKdV) equation

$$
\begin{aligned}
\frac{\partial n}{\partial \tau} & +a \mathrm{P} \int_{-\infty}^{\infty} \frac{\partial n}{\partial \xi^{\prime}} \frac{d \xi^{\prime}}{\xi-\xi^{\prime}} \\
& +b_{1} n \frac{\partial n}{\partial \xi}+b_{2} n^{2} \frac{\partial n}{\partial \xi}+c \frac{\partial^{3} n}{\partial \xi^{3}}=0
\end{aligned}
$$

where the coefficients $a$ and $c$, corresponding to the Landau damping and dispersive effects, are the same as for the KdV equation (20). The nonlinear coefficients $b_{1}$ and $b_{2}$ are, however, different and given by

$$
\begin{gathered}
b_{1}=\left(3-\sigma_{2} / \sigma_{1}^{2}\right) / 2 \sqrt{\sigma \sigma_{1}}, \\
b_{2}=(15 / 4)\left(\alpha_{2} /\left(\sigma \sigma_{1}\right)^{1 / 2}\right)\left[1-\left(\sigma_{3} / 15 \sigma_{1}^{3}\right)\right] .
\end{gathered}
$$

Here, the expressions for $\sigma_{1}$ and $\sigma_{2}$ are given before and $\sigma_{3}=\left(\sigma^{3}+\mu\right) / \sigma^{3}(1+\mu)$. If we neglect the strength of the Landau damping i.e., if we set $\alpha_{1}=0$, the term $\propto a$ in Eq. (87) vanishes, and Eq. (87) reduces to the usual $\mathrm{KdV}-\mathrm{mKdV}$ or Gardner equation for the propagation of DASWs in dusty bi-ion plasmas. Equation (87) is the required evolution equation for the first-order dust density perturbations when the plasma parameters $\sigma$ and $\mu$ assume values close to the critical values, i.e., $R_{1} \sim o(\epsilon)$. In fact, this equation is well applicable to DASWs when the plasma parameters assume the critical values or are very close to or around the critical values of $\sigma$ and $\mu$.

On the other hand, when $R_{1}=0$, i.e., when the parameters $\sigma$ and $\mu$ assume the critical values along the thin (or black) curve as in Fig. 1 1 the evolution of DASWs will no longer be describable by the KdV equation (20), but the $\mathrm{mKdV}$ equation, which can be obtained by setting $b_{1}=0$ in Eq. (87) as

$$
\frac{\partial n}{\partial \tau}+a \mathrm{P} \int_{-\infty}^{\infty} \frac{\partial n}{\partial \xi^{\prime}} \frac{d \xi^{\prime}}{\xi-\xi^{\prime}}+b_{2} n^{2} \frac{\partial n}{\partial \xi}+c \frac{\partial^{3} n}{\partial \xi^{3}}=0,
$$

where the coefficients $a, b_{2}$ and $c$ are the same as in the Gardner equation (87). We remind here that the evolution of DASWs with the effects of Landau damping are governed by the $\mathrm{KdV}, \mathrm{mKdV}$ or Gardner equations depending on whether the density ratio $\mu$ and the temperature ratio $\sigma$ assume their values well below the critical values, very close to the critical values or near, around and exactly the critical values of $\mu$ and $\sigma$ along the curve in Fig. 1 Note that the mKdV equation is obtained on the assumption that $R_{1}=0$. However, when $R_{2} \equiv 1-\sigma_{3} / 15 \sigma_{1}^{3}=0$, i.e., the nonlinear term $b_{2}$ in the $\mathrm{mKdV}$ equation vanishes in the $\sigma-\mu$ plane (See the thick or red curve in Fig. 11), then the mKdV equation fails to describe the evolution of DASWs. From Fig. 1, it is also clear that there are some values of $\sigma$ and $\mu$ which may be closer to both the curves (e.g., the lower parts of the curves for $R_{1}=0$ and $R_{2}=0$ below the point of intersection). In these parameter regimes both the KdV and $\mathrm{mKdV}$ equations may not be appropriate for the description of DASWs in plasmas. Thus, the Gardner equation, being the generalized form of $\mathrm{KdV}$ and $\mathrm{mKdV}$, describes the evolution of DASWs in dusty bi-ion plasmas for a wide range of values of $\sigma$ and $\mu$ including the critical values as in Fig. 1 .

\section{LANDAU DAMPING RATE}

To obtain the Landau damping rate for DASWs we take the Fourier transform of Eq. (87) with $b_{1}, b_{2}$ and $c$ being set to zero, according to the formula (39), and use the result that the inverse transform of $i \operatorname{sgn}(k)$ is $-(1 / \pi) \mathrm{P}(1 / \xi)$. Thus, we obtain the Landau damping rate as [21] $|\gamma|=\pi a$. This damping rate is shown in Fig. 3 with respect to the temperature ratio $\sigma$, and for different values of the density ratio $\mu$ and the mass ratio $m$. We find that for dusty plasmas with equal mass of positive ions, the value of $|\gamma|$ decreases (increases) with an increasing value of the parameter $\sigma(\mu)$. However, the case with unequal mass of ions $(m>1)$ is quite distinctive. In this case, the damping rate initially increases as long as $1<\sigma<5$, and for $\sigma>5$, the behaviors of $|\gamma|$ remain similar to the case of equal mass of ions. Thus, in contrast to dusty pair-ion plasmas 21] or dusty electron-ion plasmas, an enhancement of the hot ion concentration (or a reduction of the number of cold ions into the dust grain surface in order to maintain the charge neutrality), increases the Landau damping rate in dusty bi-ion plasmas.

\section{SOLITONS WITH LANDAU DAMPING}

We first obtain an approximate analytic soliton solution of the Gardner equation 87) with a small effect of the Landau damping. Here, we assume that $1 \sim b_{1}, b_{2} \sim c \gg a \gg \epsilon$. This can easily be verified by considering dusty plasma parameters satisfying $\sigma, \mu>1$. 


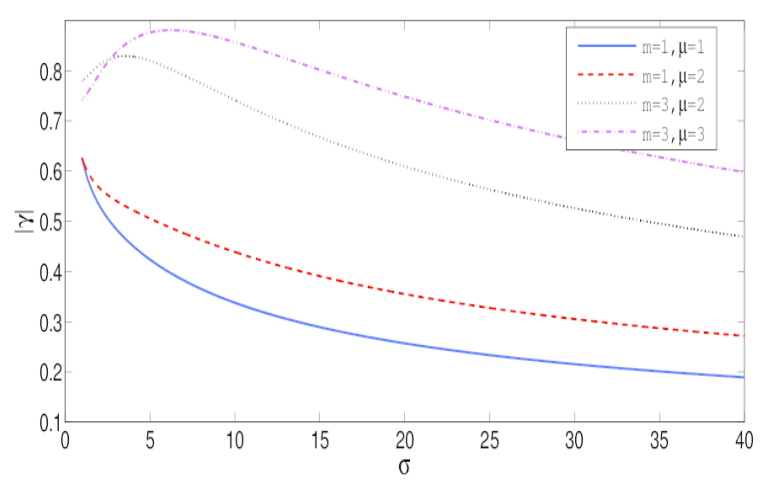

FIG. 3. The linear Landau damping rate $|\gamma|=\pi a$ is plotted against the temperature ratio $\sigma$ for different values of the mass and the density ratios $m$ and $\mu$ as in the figure.

Now, in absence of the Landau damping Eq. (87) reduces to

$$
\partial_{\tau^{\prime}} n+b_{1}^{\prime} n \partial_{\xi} n+b_{2}^{\prime} n^{2} \partial_{\xi} n+\partial_{\xi}^{3} n=0,
$$

where we have rescaled the time variable $\tau$ and the coefficients as $\tau^{\prime}=\tau c, b_{1}^{\prime}=b_{1} / c, b_{2}^{\prime}=b_{2} / c$. A traveling wave solution of Eq. (91) can be obtained as 25.

$$
n\left(\xi, \tau^{\prime}\right)=6 \bar{U}\left[b_{1}^{\prime} \pm \bar{N} \cosh \left(\left(\xi-\bar{U} \tau^{\prime}\right) / \bar{W}\right)\right]^{-1},
$$

where $\bar{W}=\sqrt{6 b_{2}^{\prime} /\left(\bar{N}^{2}-b_{1}^{\prime 2}\right)}$ is the constant width and $\bar{U}=\left(\bar{N}^{2}-b_{1}^{\prime 2}\right) / 6 b_{2}^{\prime}$ is the constant phase speed (normalized by $c_{s}$ ) of the DASWs. It can easily be verified that Eq. (87) conserves the total number of particles and an initial perturbation of the form 92 decays to zero (See for some details, e.g., Ref. 21). The latter implies that the wave amplitude $\bar{N}$ is not a constant but decreases slowly with time. Thus, we introduce a new space coordinate in a frame moving with the solitary wave and normalized to its width as 21]

$$
z=\sqrt{\left(\bar{N}^{2}-b_{1}^{\prime 2}\right) / 6 b_{2}^{\prime}}\left(\xi+\frac{b_{1}^{\prime 2}}{3 b_{2}^{\prime}} \tau^{\prime}-\frac{1}{6 b_{2}^{\prime}} \int_{0}^{\tau^{\prime}} \bar{N}^{2} d \tau^{\prime}\right),
$$

where $\bar{N}=\bar{N}\left(a, \tau^{\prime}\right)$ is assumed to vary slowly with time and $n=n\left(z, \tau^{\prime}\right)$. Under this transformation Eq. (87) reduces to

$$
\begin{aligned}
& \partial_{\tau^{\prime}} n+\left[-A+B z\left(d \bar{N} / d \tau^{\prime}\right)\right] \partial_{z} n+\left(b_{1}^{\prime} / \bar{W}\right) n \partial_{z} n \\
& +\left(b_{2}^{\prime} / \bar{W}\right) n^{2} \partial_{z} n+\left(1 / \bar{W}^{3}\right) \partial_{z}^{3} n \\
& +\left(a^{\prime} / \bar{W}\right) P \int_{-\infty}^{\infty} \partial_{z}^{\prime} n \frac{d z^{\prime}}{z-z^{\prime}}=0
\end{aligned}
$$

where $A=\left(\bar{N}^{2}-b_{1}^{\prime 2}\right) / 6 b_{2}^{\prime} \bar{W}, B=\bar{N} /\left(\bar{N}^{2}-b_{1}^{\prime 2}\right), a^{\prime}=a / c$ and we have used $\partial n / \partial z^{\prime}=\partial n / \partial z$ at $z=z^{\prime}$.
In what follows, we investigate the solution of Eq. 94, and introduce a multiple time scale analysis with respect to $a^{\prime}$. Thus, we write [21, 26]

$$
n\left(z, \tau^{\prime}\right)=n^{(0)}+a^{\prime} n^{(1)}+a^{\prime 2} n^{(2)}+a^{\prime 3} n^{(3)}+\cdots,
$$

where $n^{(i)}, \quad i=0,1,2,3, \cdots$ are functions of $\tau^{\prime}=\tau_{0}, \tau_{1}, \tau_{2}, \tau_{3}, \cdots$ in which $\tau_{i}=a^{\prime i} \tau^{\prime}$. So, we have $\partial n^{(0)} / \partial \tau^{\prime}=\left(\partial n^{(0)} / \partial \tau_{0}\right)\left(\partial \tau_{0} / \partial \tau^{\prime}\right)+$ $\left(\partial n^{(0)} / \partial \tau_{1}\right)\left(\partial \tau_{1} / \partial \tau^{\prime}\right)+\cdots, \quad \partial n^{(1)} / \partial \tau^{\prime}=$ $\left(\partial n^{(1)} / \partial \tau_{0}\right)\left(\partial \tau_{0} / \partial \tau^{\prime}\right)+\left(\partial n^{(1)} / \partial \tau_{1}\right)\left(\partial \tau_{1} / \partial \tau^{\prime}\right)+\cdots$ etc. and the similar expression for $\partial \bar{N} / \partial \tau$. Substituting the expansion (95) into Eq. (94) and using the expressions for $\partial n^{(0)} / \partial \tau^{\prime}, \partial n^{(1)} / \partial \tau^{\prime}$ and $\partial \bar{N} / \partial \tau^{\prime}$ we obtain

$$
\begin{aligned}
& \left(\partial_{\tau^{\prime}} n^{(0)}+a^{\prime} \partial_{\tau_{1}} n^{(0)}+\cdots\right)+a^{\prime}\left(\partial_{\tau^{\prime}} n^{(1)}+a^{\prime} \partial_{\tau_{1}} n^{(1)}+\cdots\right) \\
& +\left[-A+B z\left(\partial_{\tau^{\prime}} \bar{N}+a^{\prime} \partial_{\tau_{1}} \bar{N}+\cdots\right)\right] \times \\
& \partial_{z}\left(n^{(0)}+a^{\prime} n^{(1)}+\cdots\right)+(1 / \bar{W})\left(b_{1}^{\prime}+b_{2}^{\prime} n^{(0)}\right) n^{(0)} \partial_{z} n^{(0)} \\
& +\frac{a^{\prime}}{\bar{W}}\left[b_{1}^{\prime} \partial_{z}\left(n^{(1)} n^{(0)}\right)+b_{2}^{\prime} n^{(0)}\left(n^{(0)} \partial_{z} n^{(1)}+2 n^{(1)} \partial_{z} n^{(0)}\right)\right] \\
& +\left(1 / \bar{W}^{3}\right) \partial_{z}^{3} n^{(0)}+a^{\prime}\left(1 / \bar{W}^{3}\right) \partial_{z}^{3} n^{(1)} \\
& +a^{\prime} \frac{1}{\bar{W}} P \int_{-\infty}^{\infty} \partial_{z}^{\prime} n^{(0)} \frac{d z^{\prime}}{z-z^{\prime}}+\cdots=0,
\end{aligned}
$$

Equating the coefficients of the zeroth and first-order of $a^{\prime}$, we successively obtain from Eq. (96) as

$$
\begin{gathered}
\beta\left[\partial_{\tau^{\prime}}+(B z) \partial_{\tau^{\prime}} \bar{N} \partial_{z}\right] n^{(0)}+M_{0} \partial_{z} n^{(0)}=0, \\
\beta\left[\partial_{\tau^{\prime}}+(B z) \partial_{\tau^{\prime}} \bar{N} \partial_{z}\right] n^{(1)}+\partial_{z} M_{0} n^{(1)}=\beta R n^{(0)},
\end{gathered}
$$

where $\beta=\bar{W}^{3}$ and

$$
\begin{array}{r}
M_{0}=\partial_{z}^{2}+\left[n^{(0)}\left(b_{1}^{\prime}+b_{2}^{\prime} n^{(0)}\right)(A \bar{W})^{-1}-1\right], \\
R n^{(0)}=-\left[\partial_{\tau_{1}} n^{(0)}+(B z) \partial_{\tau_{1}} \bar{N} \partial_{z} n^{(0)}\right. \\
\left.+\frac{1}{\bar{W}} P \int_{-\infty}^{\infty} \partial_{z}^{\prime} n^{(0)} \frac{d z^{\prime}}{z-z^{\prime}}\right] .
\end{array}
$$

Using the boundary conditions, namely, $n^{(0)}, \partial n^{(0)} / \partial z$, $\partial^{2} n^{(0)} / \partial z^{2}, \partial^{3} n^{(0)} / \partial z^{3} \longrightarrow 0$ as $z \rightarrow \pm \infty$ it can be shown that $n^{(0)}=6 U_{0} /\left(b_{1}^{\prime} \pm \bar{N} \cosh z\right)$ is a soliton solution of the equation $M_{0}\left(\partial n^{(0)} / \partial z\right)=0$. Thus, $n^{(0)}=$ $6 U_{0} /\left(b_{1}^{\prime} \pm \bar{N} \cosh z\right)$ will be a soliton solution of Eq. (97) if and only if $\partial \bar{N} / \partial \tau=0$. So, Eq. (98) reduces to

$$
\beta\left[\partial_{\tau} n^{(1)}\right]+\partial_{z} M_{0} n^{(1)}=\beta R n^{(0)} .
$$

Next, for the existence of a solution of Eq. 101), $R n^{(0)}$ 


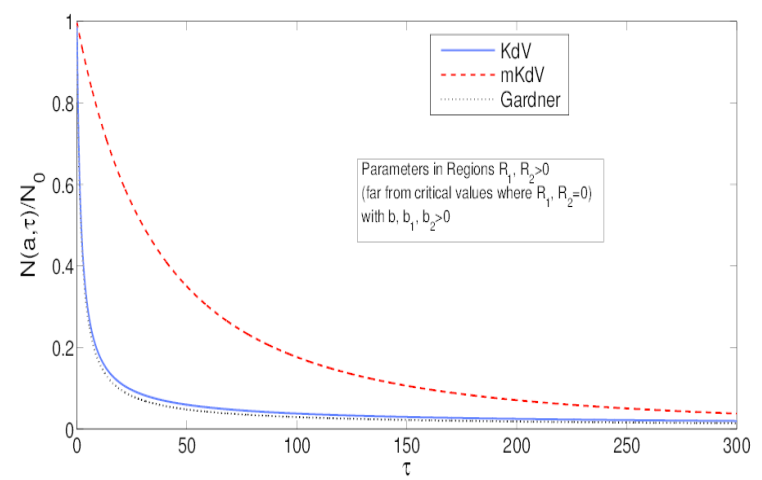

(a)

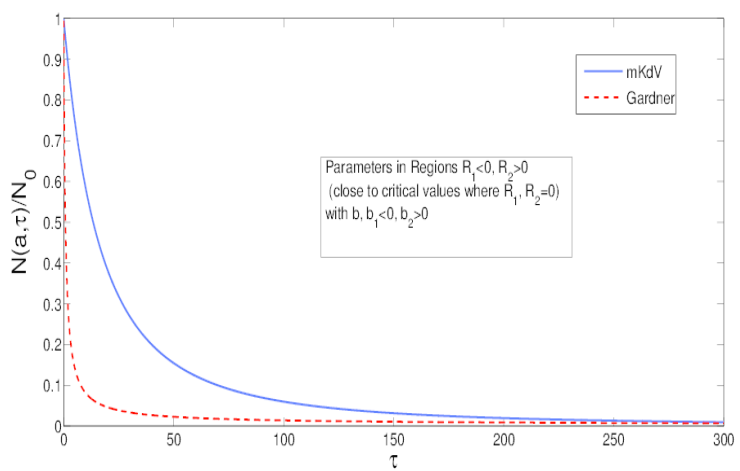

(b)

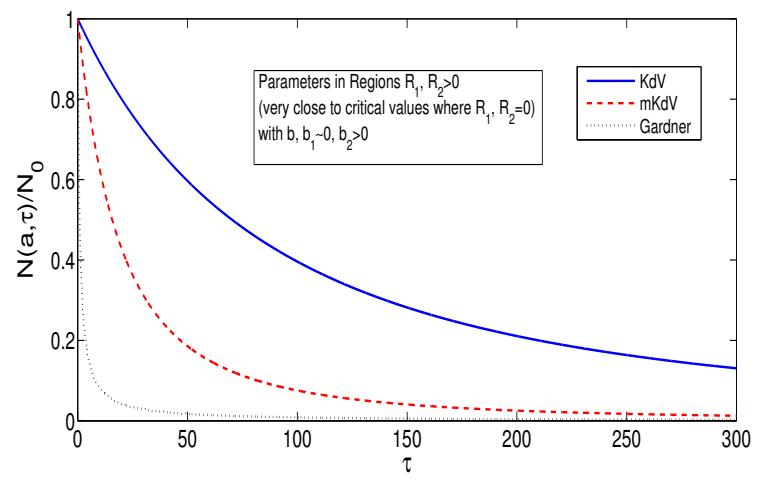

(c)

FIG. 4. The solitary wave amplitudes $N(a, \tau)$ (normalized to their equilibrium values $N_{0}$ ) with the effect of Landau damping are shown for $\mathrm{KdV}, \mathrm{mKdV}$ and Gardner solitons [Eqs. [119, 122, [112]. Subfigure 4(a) shows that when $\mu$ and $\sigma$ assume values $\left(m=\sigma=3, \mu=7.4\right.$ ) well below the critical values on the curve $R_{1}=0$ and fall in the region $R_{1}>0$, the decay rates of the amplitudes of the KdV and Gardner solitons with respect to $\tau$ are approximately the same as expected. However, the rate for the $\mathrm{mKdV}$ soliton is quite higher than the $\mathrm{KdV}$ and Gardner solitons. For values of $\mu$ and $\sigma(m=3, \sigma=15, \mu=4.5)$ close to the critical values, but in the regions $R_{1}, R_{2}<0$ for which $b, b_{1}<0$ (and hence KdV equation fails to govern the dynamics of dust-acoustic solitons), subfigure 4(b) shows that the gap between the decay rates of the Gardner and mKdV solitons becomes lower than that in Fig. 4(a) On the other hand, when $\mu$ or $\sigma$ values $(m=3, \sigma=11, \mu=5.3)$ are very close to the critical values, but still in the regions $R_{1}, R_{2}>0$ for which $b, b_{1} \approx 0$ and $b_{2}>0$, subfigure 4 (c) shows that the gaps among the amplitudes of the $\mathrm{KdV}, \mathrm{mKdV}$ and Gardner solitons again increase, and Gardner equation seems to be more appropriate for the description of dust-acoustic solitons.

must be orthogonal to all solutions $g(z)$ of $L^{+}[g]=0$ which satisfy $g( \pm \infty)=0$, where $L^{+}$is the operator adjoint to $L \equiv(\partial / \partial z) \bar{M}$, defined by

$$
\int_{-\infty}^{\infty} \psi_{1}(z) L\left[\psi_{2}(z)\right] d z=\int_{-\infty}^{\infty} \psi_{2}(z) L^{+}\left[\psi_{1}(z)\right] d z,
$$

where $\psi_{1}( \pm \infty)=\psi_{2}( \pm \infty)=0$, the operator $L^{+}$is given by

$$
L^{+} \equiv \frac{\partial^{3}}{\partial z^{3}}+\left[\frac{6 \bar{N}\left(\bar{N} \pm b_{1}^{\prime} \cosh z\right)}{\left(b_{1}^{\prime} \pm \bar{N} \cosh z\right)^{2}}-1\right] \frac{\partial}{\partial z},
$$

and the only solution of $L^{+}[g]=0$ with $g( \pm \infty)=0$ is $g(z)=\left(\bar{N}^{2}-b_{1}^{\prime 2}\right) / \bar{N}\left(b_{1}^{\prime} \pm \bar{N} \cosh z\right)$. Thus, we obtain

$$
\int_{-\infty}^{\infty} \frac{1}{\bar{N}}\left(\bar{N}^{2}-b_{1}^{\prime 2}\right)\left(b_{1}^{\prime} \pm \bar{N} \cosh z\right)^{-1} R n^{(0)} d z=0 .
$$

Substituting the expression for $R n^{(0)}$ from Eq. 100 into Eq. (104), we get 


$$
\begin{aligned}
& \pm \frac{\partial \bar{N}}{\partial \tau_{1}} \int_{-\infty}^{\infty}\left[\frac{\left(\bar{N}^{2}-b_{1}^{\prime 2}\right)}{\bar{N}^{2}} \frac{\bar{N} \cosh z}{\left(b_{1}^{\prime} \pm \bar{N} \cosh z\right)^{3}}+\frac{\bar{N} z \sinh z}{\left(b_{1}^{\prime} \pm \bar{N} \cosh z\right)^{3}}\right] d z \\
& -\frac{\left(\bar{N}^{2}-b_{1}^{\prime 2}\right)}{\bar{N} \bar{W}} \mathrm{P} \int_{-\infty}^{\infty} \int_{-\infty}^{\infty} \frac{1}{\left(b_{1}^{\prime} \pm \bar{N} \cosh z\right)} \frac{\partial}{\partial z^{\prime}}\left[\frac{1}{\left(b_{1}^{\prime} \pm \bar{N} \cosh z^{\prime}\right)}\right] \frac{d z d z^{\prime}}{z-z^{\prime}}=0
\end{aligned}
$$

from which we obtain after few steps the following

$$
\begin{aligned}
& \frac{\partial \bar{N}}{\partial \tau_{1}}\left[\frac{\left(3 \bar{N}^{2}-2 b_{1}^{\prime 2}\right)}{2 \bar{N}^{2}} \int_{-\infty}^{\infty} \frac{d z}{\left(b_{1}^{\prime} \pm \bar{N} \cosh z\right)^{2}}-b_{1}^{\prime} \frac{\left(\bar{N}^{2}-b_{1}^{\prime 2}\right)}{\bar{N}^{2}} \int_{-\infty}^{\infty} \frac{d z}{\left(b_{1}^{\prime} \pm \bar{N} \cosh z\right)^{3}}\right] d z \\
& -\frac{\left(\bar{N}^{2}-b_{1}^{\prime 2}\right)^{3 / 2}}{\bar{N} \sqrt{6 b_{2}^{\prime}}} \mathrm{P} \int_{-\infty}^{\infty} \int_{-\infty}^{\infty} \frac{1}{\left(b_{1}^{\prime} \pm \bar{N} \cosh z\right)} \frac{\partial}{\partial z^{\prime}}\left[\frac{1}{\left(b_{1}^{\prime} \pm \bar{N} \cosh z^{\prime}\right)}\right] \frac{d z d z^{\prime}}{z-z^{\prime}}=0
\end{aligned}
$$

$$
\begin{aligned}
& \int_{-\infty}^{\infty}\left(b_{1}^{\prime} \pm \bar{N} \cosh z\right)^{-2} d z=\left[2 /\left(\bar{N}^{2}-b_{1}^{\prime 2}\right)^{3 / 2}\right]\left[2 b_{1}^{\prime} \tan ^{-1}\left(\left(b_{1}^{\prime} \mp \bar{N}\right) / \sqrt{\bar{N}^{2}-b_{1}^{\prime 2}}\right)+\sqrt{\bar{N}^{2}-b_{1}^{\prime 2}}\right] \\
& \int_{-\infty}^{\infty}\left(b_{1}^{\prime} \pm \bar{N} \cosh z\right)^{-3} d z=\left[2 /\left(\bar{N}^{2}-b_{1}^{\prime 2}\right)^{5 / 2}\right]\left[\left(2 b_{1}^{\prime}+\bar{N}^{2}\right) \tan ^{-1}\left(\left(b_{1}^{\prime} \mp \bar{N}\right) / \sqrt{\bar{N}^{2}-b_{1}^{\prime 2}}\right)+\frac{3 b_{1}^{\prime}}{2}\right]
\end{aligned}
$$

Finally, using the results in Eqs. 107 and 108, we obtain from Eq. 106 as

$$
\begin{aligned}
& \frac{\partial \bar{N}}{\partial \tau_{1}} \lambda_{1}-\lambda_{2} \mathrm{P} \int_{-\infty}^{\infty} \int_{-\infty}^{\infty} \frac{1}{\left(b_{1}^{\prime} \pm \bar{N} \cosh z\right)} \times \\
& \partial_{z}^{\prime}\left(b_{1}^{\prime} \pm \bar{N} \cosh z^{\prime}\right)^{-1}\left[d z^{\prime} /\left(z-z^{\prime}\right)\right] d z=0
\end{aligned}
$$

where $\lambda_{1}$ and $\lambda_{2}$ are given by

$$
\begin{gathered}
\lambda_{1}=\left[1 /\left(\bar{N}^{2}-b_{1}^{\prime 2}\right)^{3 / 2}\right]\left[8 b_{1}^{\prime} \tan ^{-1}\left(\left(b_{1}^{\prime} \mp \bar{N}\right) / \sqrt{\bar{N}^{2}-b_{1}^{\prime 2}}\right)\right. \\
\left.+\left(\left(3 \bar{N}^{2}+b_{1}^{\prime 2}\right) / \bar{N}^{2}\right) \sqrt{\bar{N}^{2}-b_{1}^{\prime 2}}\right], \\
\lambda_{2}=\left[\left(\bar{N}^{2}-b_{1}^{\prime 2}\right)^{3 / 2} / \bar{N} \sqrt{6 b_{2}^{\prime}}\right] .
\end{gathered}
$$

Equation 109 is a first-order (in $\tau$ ) partial differential equation for the solitary wave amplitude $\bar{N}(a, \tau)$ whose exact analytic solution is much complicated. However, one can consider some particular cases as follows:

We note that for small-amplitude perturbations $\epsilon\left|n_{1}(\xi, \tau)\right| \ll 1$. However, inspecting on the solution (92) of Eq. 87 in absence of the Landau damping effect, we find that this condition may be satisfied when either $\bar{U}$ is small or the denominator of the expression on the right-hand side becomes larger. Also, for real values of $\bar{W}$ and $\bar{U}(>0)$, we have $\bar{N}^{2}>b_{1}^{\prime 2}$ when $\bar{N}$ is independent on time $\tau$. However, when $\bar{N} \equiv \bar{N}(a, \tau)$, we must have $\bar{N}^{2}(a, \tau)>b_{1}^{\prime 2} \forall \tau$ for $\lambda_{1}$ and $\lambda_{2}$ to be real. Here, we consider the case in which $\bar{N}^{2}(a, \tau) \gg b_{1}^{\prime 2} \forall \tau$. In this case, though some generalization may be lost, the basic qualitative features [21] of the solution of Eq. 109] will remain the same. Under this approximation the solution of Eq. 109 is given by a quadratic equation in $\bar{N}$ which yields two solutions (for $\zeta= \pm 1$ )

$$
\bar{N}(a, \tau)=\left(3 b_{2} / c\right)^{1 / 4} \bar{N}_{0}\left(\lambda_{3} / \lambda_{4}\right),
$$

where $\bar{N}=\bar{N}_{0}$ at $\tau=0$ and

$$
\begin{gathered}
\lambda_{3}=3^{5 / 4}\left(b_{2} / c\right)^{1 / 4} \bar{N}_{0}+\zeta\left[\left(3 b_{2} / c\right)^{1 / 2}\right. \\
\left.\times\left(3 \bar{N}_{0} \mp \pi b_{1} / c\right)^{2} \pm 2 \sqrt{2} \pi b_{1} A^{\prime} a \bar{N}_{0}^{2} \tau / c\right]^{1 / 2}, \\
\lambda_{4}=\sqrt{3 b_{2} / c}\left(6 \bar{N}_{0} \mp 2 \pi b_{1} / c\right)-\sqrt{2} A^{\prime} a \bar{N}_{0}^{2} \tau, \\
A^{\prime}=\mathrm{P} \int_{-\infty}^{\infty} \int_{-\infty}^{\infty} \operatorname{sech} z \frac{\partial}{\partial z^{\prime}}\left(\operatorname{sech} z^{\prime}\right) \frac{d z^{\prime}}{z-z^{\prime}} d z .
\end{gathered}
$$


We mention that the upper and lower signs in the expressions for $\lambda_{1}, \lambda_{3}$ and $\lambda_{4}$ are corresponding to \pm in the Gardner solution (92). In our numerical investigation, we will, however, consider $\zeta=1$ and the lower (upper) sign for the existence of solitons in different regions as in Fig. 4(a) (Figs. 4(b) and 4(c) to be shown shortly. Clearly, a decrement of the wave amplitude of the Gardner soliton is seen to occur.

On the other hand, in absence of the Landau damping effects (i.e., when $a=0$ ), the $\mathrm{KdV}$ and $\mathrm{mKdV}$ equations [Eqs. 20 and (90)] have the following traveling wave solutions

$$
\begin{aligned}
& n=N \operatorname{sech}^{2}[(\xi-U \tau) / W], \\
& n=\widetilde{N} \operatorname{sech}[(\xi-\widetilde{U} \tau) / \widetilde{W}],
\end{aligned}
$$

where $N=3 U / b$ and $\tilde{N}=\sqrt{6 \widetilde{U} / b_{2}^{\prime}}$ are the constant amplitudes, $W=\sqrt{12 c / N b} \equiv \sqrt{4 c / U}$ and $\widetilde{W}=\sqrt{1 / \widetilde{U}}$ are the constant widths, and $U$ and $\widetilde{U}$ are the constant phase speeds (normalized by $c_{s}$ ) of the $\mathrm{KdV}$ and $\mathrm{mKdV}$ solitons.

Following the same procedure as above and assuming that is a small parameter and $1 \sim b, b_{2} \sim c \gg a \gg$ $\epsilon$, analytic solutions of Eqs. 20 and (90) can also be obtained. Thus, for the KdV equation (20) we have the following solution [21]

$$
n=N(a, \tau) \operatorname{sech}^{2}\left[\left(\xi-\frac{b}{3} \int_{0}^{\tau} N d \tau\right) / W\right]+o(a)
$$

where

$$
N(a, \tau)=N_{0}\left[1+\left(\tau / \tau_{0}\right)\right]^{-2}
$$

with $N=N_{0}$ at $\tau=0$ and $\tau_{0}$ being given by

$$
\tau_{0}^{-1}=\frac{a}{4} \sqrt{\frac{b N_{0}}{3 c}} \mathrm{P} \int_{-\infty}^{\infty} \int_{-\infty}^{\infty} \frac{\operatorname{sech}^{2} z}{z-z^{\prime}} \frac{\partial}{\partial z^{\prime}}\left(\operatorname{sech}^{2} z^{\prime}\right) d z d z^{\prime}
$$

Also, for the mKdV equation (90), a substitution of $b_{1}=$ 0 in Eqs. 109-(111) gives rise the following solution

$$
n=\tilde{N}(a, \tau) \operatorname{sech}\left[\left(\xi-\frac{1}{6 b_{2}^{\prime}} \int_{0}^{\tau^{\prime}} \widetilde{N}^{2} d \tau^{\prime}\right) / \widetilde{W}\right]+o(a)
$$

where

$$
\widetilde{N}(a, \tau)=\widetilde{N}_{0}[1-(\tau / \widetilde{\tau})]^{-2},
$$

with $\tilde{N}=\widetilde{N}_{0}$ at $\tau=0$ and $\widetilde{\tau}$ being given by

$$
\tilde{\tau}^{-1}=\frac{a \tilde{N}_{0}}{3 \sqrt{6 b_{2}^{\prime}}} \mathrm{P} \int_{-\infty}^{\infty} \int_{-\infty}^{\infty} \frac{\operatorname{sech} z}{z-z^{\prime}} \frac{\partial}{\partial z^{\prime}}\left(\operatorname{sech} z^{\prime}\right) d z d z^{\prime}
$$

We numerically investigate the time variations of the wave amplitudes of the $\mathrm{KdV}, \mathrm{mKdV}$ and Gardner solitons given by Eqs. 118, 121 and 112 respectively. The results are shown in Fig. 4 for different parameter regimes as applicable for the existence of $\mathrm{KdV}, \mathrm{mKdV}$ and Gardner solitons. We note that when the values of $\sigma$ and $\mu$ fall in the common regions of $R_{1}, R_{2}>0$ (for which $b, b_{1}$ and $b_{2}>0$ ), but well below the critical values as in Fig. 1, the KdV equation suffices to describe the nonlinear evolution of DA solitons. This is evident from Fig. 4(a). Here, it is seen that the behaviors of the decay rates of the $\mathrm{KdV}$ and Gardner solitons are almost similar having their values close to each other (See the solid and dotted lines), however, the rate becomes higher (in magnitude) for the $\mathrm{mKdV}$ solitons. Also, the $\mathrm{KdV}$ soliton points to lower wave amplitude than the $\mathrm{mKdV}$ and Gardner solitons at large $\tau$. Thus, in the parameter regimes with $\sigma$ and $\mu$ far below the critical values, mKdV equation may not be relevant, however, the $\mathrm{KdV}$ and Gardner equations can give rise to approximately the same results for the evolution of DA solitons in plasmas. When values of $\sigma$ and $\mu$ are close to the critical values but in the regions $R_{1}<0, R_{2}>0$ (for which $b, b_{1}<0$ and $b_{2}>0$ ), the $\mathrm{KdV}$ solitons cease to exist (because $b$ must be positive for $\tau_{0}$ to be real). In this case, equations involving higher-order nonlinearities, namely Gardner or mKdV equation is necessary for the evolution of DA solitons. Figure 4(b) shows that the Gardner equation is more appropriate than the $\mathrm{mKdV}$ equation as it gives rise to solitons with lower amplitudes than the mKdV solitons. On the other hand, when $\sigma$ and $\mu$ assume values very close to the critical values but still in the common regions of $R_{1}, R_{2}>0$ (for which $b, b_{1} \approx 0$ and $b_{2}>0$ ), Fig. 4(c) shows that the decay rates of the $\mathrm{KdV}$ and $\mathrm{mKdV}$ solitons get higher (in magnitude) than that of the Gardner soliton. In this case, $\mathrm{KdV}$ equation fails, however, Gardner equation gives better (than the mKdV equation) results for the properties of DA solitons with Landau damping. It is also found that (not shown in the figure) as the mass ratio $m$ increases, the amplitudes $N(a, \tau), \widetilde{N}(a, \tau)$ and $\bar{N}(a, \tau)$ for $\mathrm{KdV}, \mathrm{mKdV}$ and Gardner solitons decrease. Also, as $\sigma$ increases, both $N(a, \tau)$ and $\bar{N}(a, \tau)$ increase, but $\widetilde{N}(a, \tau)$ decreases. Furthermore, an enhancement of $N(a, \tau)$ and a decrement of $\widetilde{N}(a, \tau)$ and $\bar{N}(a, \tau)$ are seen to occur with an increase of the density ratio $\mu$.

\section{CONCLUSION}

We have investigated the nonlinear propagation of small-amplitude dust-acoustic solitary waves in a dusty plasma with the effects of Landau damping associated with the finite inertial effects of hot and cold ions. By considering the lower and higher order perturbations, the evolution of dust-acoustic solitons are shown to be governed by $\mathrm{KdV}, \mathrm{mKdV}$ or Gardner (KdV-mKdV) equation. The existence of the critical values of $\sigma$ and $\mu$ (de- 
noting, respectively, the temperature and the density ratios of hot to cold ions) in the $\sigma-\mu$ parameter plane for which the $\mathrm{KdV}$ and $\mathrm{mKdV}$ solitons cease to exist is given. It is found that the $\mathrm{KdV}$ solitons with Landau damping exist only for values of $\sigma$ and $\mu$ well below their critical values. Beyond the $\mathrm{KdV}$ limit, i.e., when $\sigma$ and $\mu$ assumes values close to their critical values, the Gardner equation is seen to be more appropriate than the $\mathrm{mKdV}$ equation for the evolution of small-amplitude dust-acoustic solitons in bi-ion dusty plasmas. It is also found that regardless of the ion masses equal or not, there exist a common value, i.e., $\sigma, \mu \approx 10$ for which both the $\mathrm{KdV}$ and $\mathrm{mKdV}$ equations fail to govern the dust-acoustic solitons in plasmas. The properties of the wave phase velocity and the Landau damping rate are also studied with different values of $\sigma, \mu$ and the mass ratio $m$ of cold to hot ions. It is shown that the damping rate is quite different for dusty plasmas with equal and unequal masses of positive ions. Distinctive features of the $\mathrm{KdV}, \mathrm{mKdV}$ and Gardner solitons with a small (but finite) effect of the Landau damping are compared for appropriate parameter values of $\sigma, \mu$ and $m$. It is seen that beyond the $\mathrm{KdV}$ limit, Gardner equation always points to smaller wave amplitudes with the effect of Landau damping. The results should be useful for understanding the collisionless wave damping of dust-acoustic solitons in laboratory as well as space plasmas such as those in planetary rings (e.g., the F-ring of Saturn) where negatively charged dusts and two species of positive ions are the major constituents.

\section{ACKNOWLEDGMENTS}

A.B. thanks University Grants Commission (UGC), Government of India, for Rajib Gandhi National Fellowship (RGNF) with Ref. No. F1-17.1/2012-13/RGNF2012-13-SC-WES-17295/(SA-III/Website).
[1] R. S. Narcisi, A. D. Bailey, L. Della Lucca, C. Sherman, and D. M. Thomas, J. Atmos. Terr. Phys. 33, 1147 (1971).

[2] A. J. Coates, F. J. Crary, G. R. Lewis, D. T. Young, J. H. Waite, Jr., and E. C. Sittler, Jr., Geophys. Res. Lett. 34, L22103, doi: 10.1029/2007GL030978 (2007).

[3] S. H. Kim and R. L. Merlino, Phys. Plasmas 13, 052118 (2006).

[4] S. H. Kim, R. L. Merlino, J. K. Meyer, and M. Rosenberg, J. Plasma Phys. 79, 1107 (2013).

[5] M. Rosenberg and R. L. Merlino, Planet. Space Sci. 55, 1464 (2007).

[6] A. P. Misra, N. C. Adhikary, and P. K. Shukla, Phys. Rev. E 86, 056406 (2012).

[7] A. P. Misra and N. C. Adhikary, Phys. Plasmas 20, 102309 (2013).

[8] M. M. Hatami, B. Shokri, and A. R. Niknam, J. Phys. D: Appl. Phys. 42, 025204 (2009).

[9] A. A. Mamun, Astrophys. Space Sci. 260, 507 (1999).

[10] C. K. Goertz, Rev. Geophys. 27, 271 (1989).

[11] G. C. Reid, J. Geophys. Res. 95, 13891 (1990).

[12] A. Barkan, R. L. Merlino, and N. DAngelo, Phys. Plasmas 2, 3563 (1995).

[13] P. R. Chalise, M. S. Rahman, H. Ghomi, Y. Hayashi, M. Watanabe, A. Okino, T. Ano, M. Shoda, and E. Hotta,
IEEE Trans. Plasma Sci. 321532 (2004).

[14] P. K. Shukla and A. A. Mamun, Introduction to Dusty Plasma Physics (Institute of Physics Publishing, Bristol, 2002).

[15] N. N. Rao, P. K. Shukla, M. Y. Yu, Planet Space Sci. 38, 543 (1990).

[16] L. Landau, J. Phys. (USSR) 10, 25 (1946).

[17] J. H. Malmberg and C. B. Wharton, Phys. Rev. Lett. 13, 184 (1964).

[18] E. Ott and R. N. Sudan, Phys. Fluids 12, 2388 (1969).

[19] E. Ott and R. N. Sudan, Phys. Fluids 13, 1432 (1970).

[20] A. Mukherjee, A. Bose, and M. S. Janaki, Phys. Plasmas 21, 072303 (2014).

[21] A. Barman and A. P. Misra, Phys. Plasmas 21, 073708 (2014).

[22] H. Zhang, Y. Yang, J. Zhang, X-R Hong, M-M Lin, L. Yang, X. Qi, and W-S Duan, Phys. Plasmas 21, 113706 (2014).

[23] M. Emamuddin and A. A. Mamun, Astrophys. Space Sci. 351, 561 (2014).

[24] T. Taniuti and N. Yajima, J. Math. Phys. 10, 1369 (1969).

[25] A-M Wazwaz, Commun. Nonlinear Sci. Numer. Simulat. 12, 1395 (2007).

[26] A. Bandyopadyay and K. P. Das, Phys. Plasmas 9, 465 (2002). 